\title{
Atom Probe Tomography of Grain Boundaries in Ion Conducting Oxides
}

\author{
David R. Diercks and Brian P. Gorman \\ Colorado School of Mines, Department of Metallurgical and Materials Engineering, Golden, CO, \\ USA
}

In ionic conductors, grain boundaries are particularly important as they frequently have conductivities at least two orders of magnitude less than the bulk [1]. Therefore, being able to quantitatively characterize the grain boundary nature to ascertain the reasons behind the decreased conductivity is indispensable for guiding future improvements. The common driving force for differences in the chemistry at the grain boundaries versus the bulk for a material is the grain boundary energy, which is governed by the enthalpy and entropy of mixing. Variables such as temperature, pressure, and relative crystallographic orientation will modify these values, possibly changing the nature of the segregation. In addition to these thermodynamic considerations, ionic materials by nature of their bonding have space-charge considerations. Calculations and electrical measurements [2] along with some microscopy [3] have demonstrated that in high purity materials, the formation of a space-charge layer around the grain boundary has a significant local effect on the structure. However, the challenge in measuring these changes, particularly oxygen concentration, over such a small region makes atom probe tomography (APT) one of the only methods available for direct quantification.

APT has been demonstrated to be an essential technique for local 3D chemical analysis of nanometersized structures. This has proven particularly useful in identifying local chemistry changes across grain boundaries. For example, APT has been used to demonstrate segregation of solutes [4-5] and impurities [6] and precipitate formation at grain boundaries [7] in metals. More recently, there have been examples of APT measurements of impurity and dopant segregation at the grain boundaries of oxide materials [8$10]$.

In this work an oxygen ion conductor $\mathrm{Ce}_{1-\mathrm{x}} \mathrm{Nd}_{\mathrm{x}} \mathrm{O}_{2-\mathrm{x} / 2}$ and a proton conductor $\mathrm{BaCe}_{0.2} \mathrm{Zr}_{0.7} \mathrm{Y}_{0.1} \mathrm{O}_{2.95}$ (BCZY27) were analysed with a combination of transmission electron microscopy (TEM) and APT with particular emphasis on analysis of the grain boundary regions. The use of TEM before and after APT allowed for direct determination of the volume analyzed for correlation with the APT data as shown in Figure 1. In the $\mathrm{Nd}$-doped ceria, segregation at the grain boundary was clearly visible and quantifiable as shown in Figure 2. The BCZY27 specimen was solid state reactive sintered using $2 \mathrm{wt} \% \mathrm{NiO}$ [11] and then operated in a reducing atmosphere for $1000 \mathrm{hrs}$. Most grain boundaries were observed to be compositionally no different than the bulk. However, as shown in Figure 3, some pockets of $\mathrm{Ni}$ enrichment were found at and near some grain boundaries.

\section{References:}

[1] Xin Guo and Rainer Waser, Prog. in Mater. Sci. 51 (2006) p. 151.

[2] X. Guo and J. Maier, J. Electrochem. Soc. 148 (2001) p. E121.

[3] Yuanyuan Lei, Yasuo Ito, and Nigel D. Browning, J. Am. Ceram. Soc., 85 (2002) p. 2359.

[4] J. A. Horton and M. K. Miller, Acta metall. 35 (1987) p. 133.

[5] B. W. Krakauer and D. N. Seidman, Rev. Sci. Instrum. 63 (1992) p. 4071. 
[6] Dieter Isheim et al, Scripta Mater. 55 (2006), p. 35.

[7] S.P. Ringer, K. Hono, and T. Sakurai, Metall. and Mater. Trans. A 26 (1995), p 2207.

[8] Y.M. Chen et al, Scripta Mater. 61 (2009) p. 693.

[9] Emmanuelle A. Marquis et al, Mater. Today 13 (2010) p. 34.

[10] F. Li et al, Scripta Mater. 63 (2010) p. 332.

[11] Coors W.G. in "Advances in Ceramics - Synthesis and Characterization, Processing and Specific Applications”, ed. Costas Sikalidis (InTech, Rijeka, Croatia) p. 479.

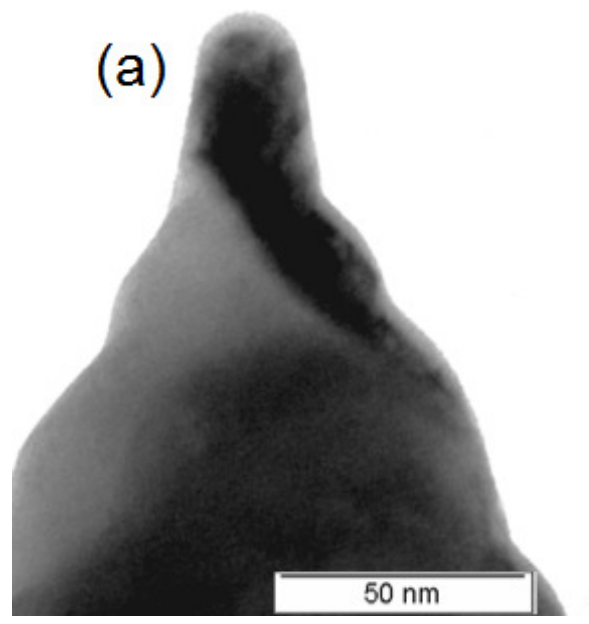

(b)

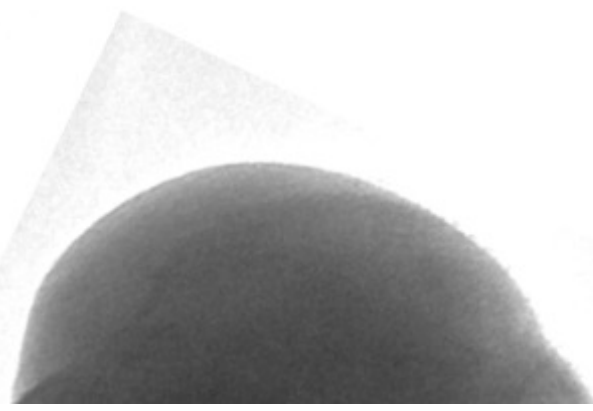

(c)

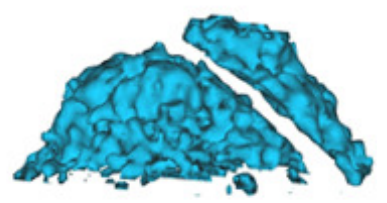

Figure 1. (a) TEM image of a $\mathrm{Ce}_{0.7} \mathrm{Nd}_{0.3} \mathrm{O}_{1.85}$ APT specimen with a grain boundary near the apex. (b) TEM image of the same specimen after APT analysis. (c) APT reconstruction using a 66 at\% oxygen isoconcentration surface. A deficiency of oxygen at the grain boundary is clearly visible.

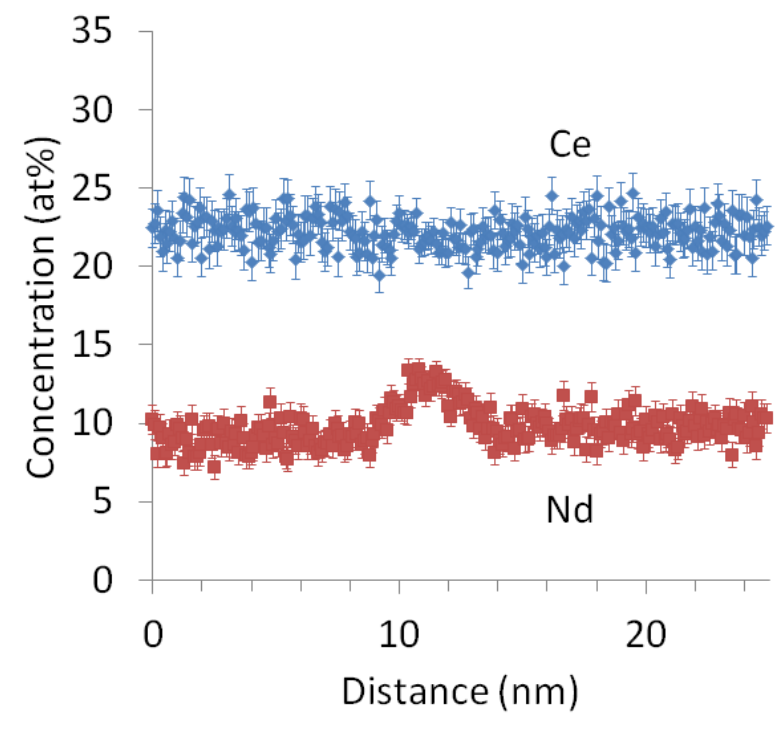

Figure 2. Concentration of $\mathrm{Ce}$, and $\mathrm{Nd}$ across a grain boundary from a $\mathrm{Ce}_{0.7} \mathrm{Nd}_{0.3} \mathrm{O}_{1.85}$ APT analysis. An increase in $\mathrm{Nd}$ concentration is apparent at the grain boundary.

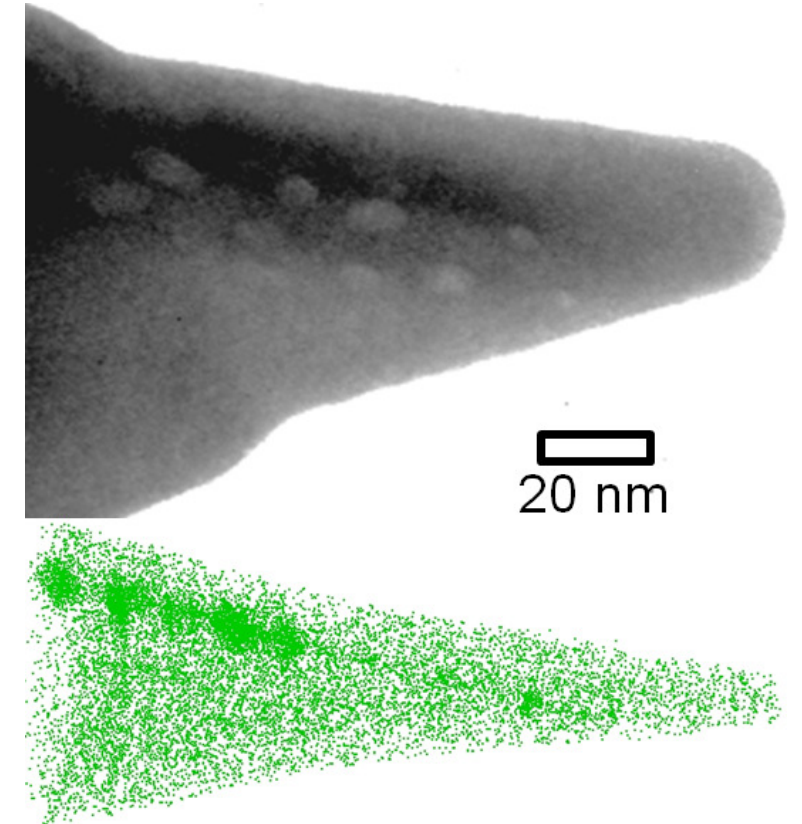

Figure 3. TEM image (top) and APT reconstruction (bottom) of BCZY27. The green points are $\mathrm{Ni}$. 